\title{
Rapid onset of ptosis indicates accurate intraconal placement during retrobulbar anaesthetic injection
}

\author{
James P Morgan, Louis G Clearkin
}

\begin{abstract}
Background-Anecdotal evidence has previously suggested that retrobulbar local anaesthetic (LA) injection is accompanied by the rapid onset of ptosis. Here the validity of this potentially valuable sign is tested.

Methods-25 patients received a retrobulbar injection and the times for development of ptosis and akinesia in other extraocular muscle groups were recorded and compared. The effects of retrobulbar injections were also studied on posterior orbital structures in 10 patients using low frequency ultrasound.

Results-The mean time of onset of ptosis was 4.76 seconds, an order of magnitude less than times recorded for akinesia in other muscle groups. Ultrasonography revealed a significant distension of the extraocular muscle cone during retrobulbar injection.

Conclusion-Ptosis develops significantly more rapidly than other motor effects in retrobulbar anaesthesia and can therefore be taken as an indicator of accurate intraconal placement. Retrobulbar injection is associated with significant distension of the extraocular muscle cone.

(Br f Ophthalmol 2001;85:363-365)
\end{abstract}

Retrobulbar local anaesthetic (LA) injection remains popular in ocular surgery ${ }^{1}$ because it

Accepted for publication 4 October 2000 provides a rapid, profound, and reliable anaesthesia and akinesia. The essential difference between retrobulbar and the newer peribulbar/ parabulbar techniques is that in retrobulbar, the anaesthetic is deposited directly within the extraocular muscle "cone". ${ }^{2}$ This allows direct access to the important motor and sensory ocular nerves. In contrast, the peribulbar technique relies on diffusion of anaesthetic throughout the orbital tissues. ${ }^{3}$ In the past it has been suggested that delivery of a retrobulbar injection is often accompanied by the almost instantaneous onset of ptosis ${ }^{45}$ and this sign has therefore been taken by some to indicate accurate intraconal placement of the anaesthetic. However, this phenomenon has never been tested experimentally. There are no reports in the literature of a similarly rapid ptosis accompanying peribulbar or parabulbar injection and our personal experiences bear this out. In this study we therefore test the validity of the above claim, by observing and timing the development of ptosis and extraocular muscle paresis after a series of retrobulbar injections. We also investigated the effects of such injections on the structures of the posterior orbit by means of ultrasound.

\section{Methods}

In all, 25 patients were selected consecutively before ocular surgery and informed consent obtained. A transconjunctival, modified Atkinson type ${ }^{2}$ retrobulbar injection was administered to each patient over 3-4 seconds. A total volume of $5.5 \mathrm{ml}$ (consisting of $2.5 \mathrm{ml}$ lignocaine $2 \%$ with $1: 200000$ adrenaline, 2.5 $\mathrm{ml}$ of $0.75 \%$ bupivacaine, and $0.5 \mathrm{ml}$ (75 Usp units) of hyaluronidase) was injected via a 30 $\mathrm{mm}, 23$ gauge needle. This moderately large volume had been the standard volume in use in our department for a number of years and had proved effective and safe. Times of onset and completion of ptosis (to the nearest second) were recorded by stopwatch. Times for paresis in the muscle groups responsible for upgaze, downgaze, abduction, and adduction were also measured (to the nearest 5 seconds) by directing patients to continually follow a fixation target, focusing with the fellow eye where necessary. Times in excess of 5 minutes were discounted because they were probably due to bulk diffusion of the LA.

A separate group of 10 patients had ultrasound performed during retrobulbar injection, to study the effects on posterior orbital structures. The same anaesthetic technique was used with the ultrasound probe placed on the patient's upper lid throughout. A visual cue (letter "O") was inserted into the real time recording sequence to indicate the start of 
injection. An "Ultramark 9" (Advanced Technology Laboratories) ultrasound scanner, emitting a $3.0 \mathrm{MHz}$ beam from a $20 \mathrm{~mm}$ diameter, phased array probe was used. This equipment is commonly used in vascular scanning. Conventional two dimensional and colour Doppler modes are available.

Data were analysed using non-parametric tests because they did not follow a strictly normal distribution. The Mann-Whitney test was used to compare times of onset of ptosis with times of paralysis in other individual muscle groups. The Kruskal-Wallis test was used for multiple group comparisons.

\section{Results}

GENERAL OBSERVATIONS

After injection, the development of ptosis followed a predictable, almost stereotypical, pattern. In all cases, ptosis causing complete or almost complete (minimum $90 \%$ ) abolition of
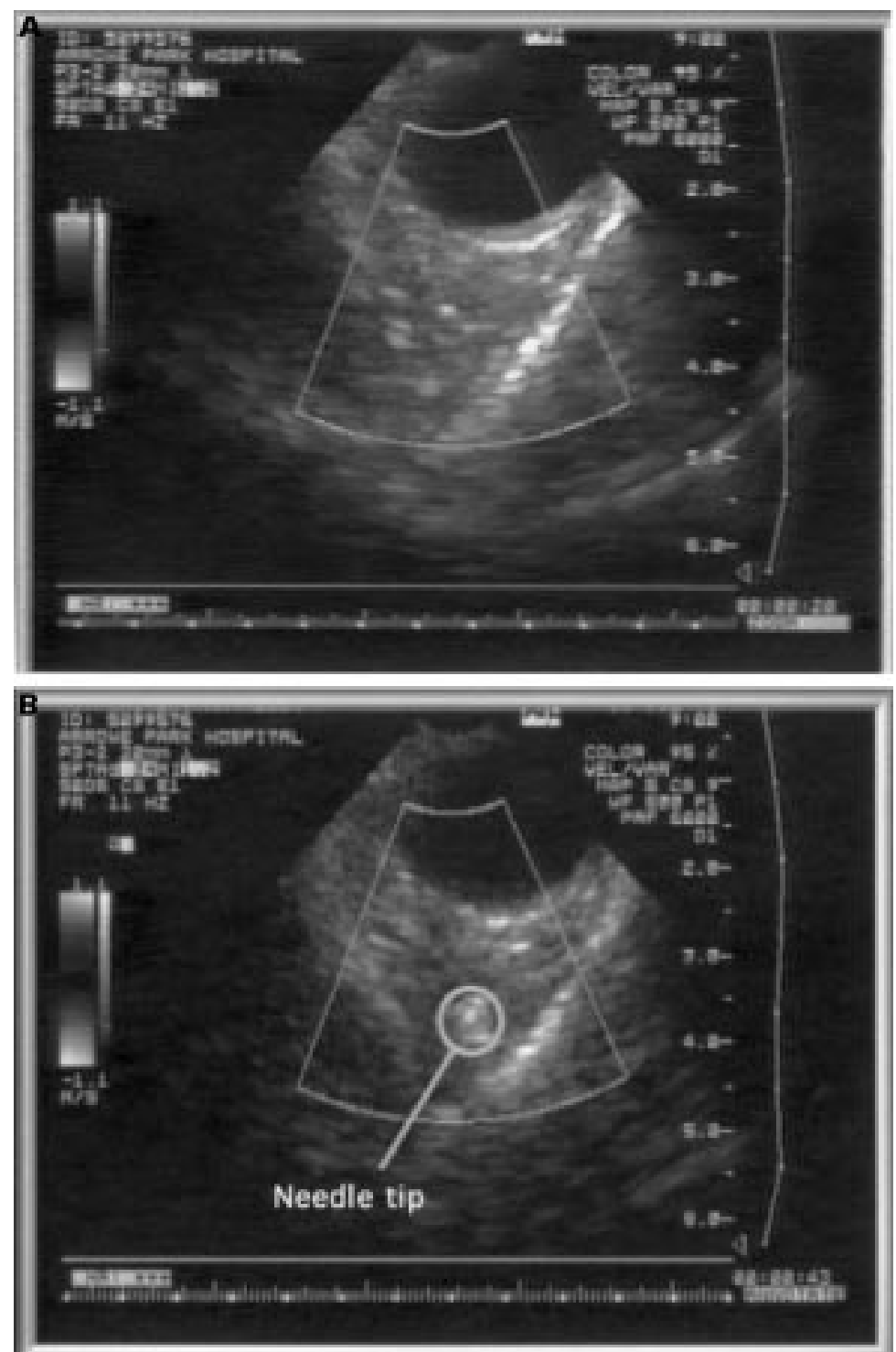

Figure 1 These ultrasound images are taken from a video recording of the orbit of a patient undergoing retrobulbar injection. The injection needle tip is clearly seen within the extraocular muscle cone. A substantial distension of the cone and anterior displacement of the globe can be seen in the later frame. (A) Posterior orbital structures immediately before injection. (B) The corresponding image at completion of injection. the palpebral fissure developed within approximately 5 seconds of its onset. Of the remaining cases of incomplete initial ptosis, some progressed slowly to complete ptosis while the remainder remained unchanged. As it was difficult to determine whether a lid was continuing to close slowly or had indeed already stopped, the times recorded for development of "maximal" ptosis are questionable. We noted that a mild proptosis often accompanied the injection.

TIMES OF EXTRAOCULAR MUSCLE PARESIS

The times of action of the retrobulbar injections on the various muscle groups are presented in Table 1. The mean time of onset of ptosis was 4.76 seconds (range 2-11, SD 1.98). This compared with a time of $82.75 \mathrm{sec}-$ onds $(20-240,56.3)$ for maximal ptosis. Other means were 66.88 seconds $(15-210,54.23)$ for abduction, 79.55 seconds $(20-225,61.1)$ for downgaze, 105.2 seconds $(20-285,82.9)$ for upgaze, and 111.1 seconds $(20-300,73.82)$ for adduction. Because values for maximal ptosis were unreliable and nearly all eyelid closure occurred shortly after the onset of ptosis, we relied on the latter value in our conclusions. Tests show this value to be statistically distinct from times recorded for the other groups $(p<0.0001)$. Differences among the latter have in turn been shown not to be statistically significant $(p=0.119)$. These other extraocular muscle groups did not succumb to the effects of injection in any recognisable pattern.

\section{ORBITAL ULTRASOUND}

The 10 ultrasound recordings of typical retrobulbar injections confirmed that the injection needle tip, where visible, was clearly within the muscle cone. It is therefore clear that the injections were indeed "retrobulbar". A reproducible, gross distension of the extraocular muscle "cone" (best appreciated on video) and slight anterior displacement of the globe were seen soon after the injection began. Representative still photographs are presented in Figure 1.

\section{Discussion}

In our study, rapid development of ptosis accompanied all retrobulbar injections. In addition, onset of ptosis preceded akinesia of all other muscle groups. This occurred several orders of magnitude more rapidly, suggesting perhaps that different mechanisms of action operate. The timings, together with ultrasonic confirmation of the intraconal nature of the injections, prove that rapid early ptosis during retrobulbar injection indicates accurate intraconal placement of the LA. Awareness of this clinical sign could help prevent the administration of unnecessary "top up" injections, as well as providing a valuable source of feedback to the anaesthetist.

The elevators of the upper lid, levator palpebrae superioris and Müller's muscle, are innervated by the distal part of the superior division of the third nerve and post-ganglionic sympathetic fibres respectively. ${ }^{6-8}$ On its own, the demonstration of microanatomical differences (such as small overall nerve diameter or 
preponderance of small diameter fibres) between the superior and inferior divisions of the third nerve would not be sufficient to explain the phenomenon of early ptosis, because superior rectus, which is also supplied by the third nerve, is initially spared. Total blockade of the small sympathetic fibres that innervate Müller's muscle would produce a ptosis equivalent to that in complete Horner's syndrome. However, as this produces at most mild to moderate ptosis $(1-4 \mathrm{~mm}),{ }^{9}{ }^{10}$ it could not be responsible for the almost complete ptosis seen immediately after retrobulbar injection.

We note with interest that the range of time over which ptosis developed (2-11 seconds) coincided closely with the time scale for distension of the extraocular muscle cone recorded on ultrasound. While it was not possible for this study to establish a causal link, we would like to point out that extraocular muscle weakness and ptosis have been induced in the past by the administration of saline retrobulbar injections. ${ }^{11}$ The possibility of a mechanical cause for the early development of ptosis in retrobulbar anaesthesia cannot therefore be discounted.

1 Leaming DV. Practice styles and preferences of ASCRS members 1998 survey. F Cataract Refract Surg 1999; 25:851-9.

2 Atkinson W. Retrobulbar injection of anesthetic within the muscle cone. Arch Ophthalmol 1936;16:494.

3 Davis DB, Mandel MR. Posterior peribulbar anesthesia: an alternative to retrobulbar anesthesia. $\mathcal{F}$ Cataract Refract Surg 1986;12:182-4.

4 Johnson RW, Forrest FC. Local and general anaesthesia for ophthalmic surgery. Oxford: Butterworth-Heinemann, phthalmic surgery. Oxford: Butterworth-Heinemann, 1994

5 Smith GB, Hamilton RC, Carr CA. Ophthalmic anaesthesia-a practical handbook. 2nd ed. London: Arnold, 1996.

6 Sunderland S, Hughes ES. The pupilloconstrictor pathway and the nerves to the ocular motor muscles in man. Brain 1946;69:301-9.

7 Sacks JG. Peripheral innervation of extraocular muscles. $\mathrm{Am}$ f Ophthalmol 1983;95:520-7.

8 Bron A, Tripathi R, Tripathi B. Wolff's anatomy of the eye and orbit. 8th ed. London: Chapman and Hall Medical, 1997.

9 Hart WM, ed. Adler's physiology of the eye. 9 ed. St Louis: Mosby Year Book, 1992.

10 Walsh TJ. Neuro-ophthalmology, clinical signs and symptoms. 4 ed. Baltimore: Williams and Wilkins, 1997.

11 Carroll F, De Roeth A. The effect of retrobulbar injections of procaine on the optic nerve. Trans Am Acad Ophthalmol Ot procaine on the optic nerve.

\section{Contributors please note:}

Communications from all countries except the UK and Republic of Ireland should be sent to Professor C Hoyt, Editor, British fournal of Ophthalmology, University of California, Department of Ophthalmology, 10 Kirkham Street, K 301, San Francisco, CA 94143-0730, USA (tel: 001415 502-6871; fax: 001415 514-1521).

Manuscripts from the UK and the Republic of Ireland should be sent to Professor Andrew Dick, UK Editor, British fournal of Ophthalmology, Division of Ophthalmology, University of Bristol, Lower Maudlin Street, Bristol BS1 2LX (tel: +44 (0) 0117 929-4496; fax: +44 (0) 117 929-4607). 